\title{
Quality of Life of Emirati Women With Breast Cancer
}

Linda Smail ( $\square$ linda.smail@zu.ac.ae)

Zayed University

Ghufran A. Jassim

Royal College of Surgeons in Ireland - Bahrain

\section{Sarah Khan}

Zayed University

Syed Tirmazy

Dubai Hospital

Mouza Al Ameri

Tawam Hospital

\section{Research Article}

Keywords: Breast Cancer, Emirati Women, Quality of Life, Health Care Policy, EORTC QLQ-BR23, EORTC QLQ-30

Posted Date: February 28th, 2022

DOI: https://doi.org/10.21203/rs.3.rs-1225528/v2

License: () (i) This work is licensed under a Creative Commons Attribution 4.0 International License. Read Full License 


\section{Abstract}

Background. This study examined the quality of life of Emirati women with breast cancer (BC).

Methods. This study was a population-based study of a random sample of 250 Emirati women with BC. Quality of life (QoL) was assessed using the European Organization for Research and Treatment of Cancer Quality of Life Cancer-Specific version (EORTC QLQ-C30, v.3.0) and the EORTC QoL Breast Cancer-Specific version (EORTC QLQ-BR23) translated into Arabic.

Results. The mean age and median age of the 250 participants were 53.4 (SD \pm 11.3 ) and 52 years, respectively.

Participants had a mean global health score of 74.73 (SD \pm 18.25 ), with a minimum of 16.67 , indicating a good level of wellbeing.

On the QLQ-C30, the Emirati women seemed to perform well on the functional scales but poorly on the symptom scales. While social functioning scored the highest $(82.33 \pm 28.38)$ among the functional scales, emotional functioning scored the lowest $(68.43 \pm 30.02)$. The most worrying symptom was sleep disturbance $(47.87 \pm 38.46)$, followed by fatigue $(38.18 \pm 30.31)$ and pain $(29.13 \pm 28.01)$. Financial impact scored the lowest, indicating that most women did not have financial issues related to their cancer.

On the QLQ-BR23, we found that participants performed above average to very good on the functional scales but poorly on the symptom scales. While sexual functioning scored the highest $(86.07 \pm 22.61)$ among the functional scales, future perspective scored the lowest $(50.80 \pm 37.92)$. The most worrying symptom was upset by hair loss $(61.01 \pm 37.35)$, followed by arm symptoms $(33.73 \pm 28.08)$.

Conclusions. Emirati BC survivors reported good QoL overall. The most bothersome symptoms were sleep disturbance, fatigue, pain, hair loss and arm symptoms. Emirati women scored average on all functional scales, which indicates mediocre functioning, but high on the symptom scales, which indicates worse symptoms. Factors associated with a decline in the domains of QoL included age, income, education, metastases, mastectomy, and lymph node dissection. Recommendations to address these issues are discussed.

\section{Trial Registration: NA}

\section{Background}

Female breast cancer (BC) is the leading cause of death among women and one of the leading cancer types worldwide in terms of the number of new cases since 2018 [1]. Many efforts have been made over the last decade not only to understand, prevent, diagnose, and treat BC but also to improve the quality of life (QoL) of BC survivors.

Surviving BC can have a very large impact on survivors' lives. People often presume that surviving the disease is the end of the battle. What they do not know is that survivors face severe challenges, both emotional and physical. Some of the symptoms usually include depression, pain, fatigue, loss of interest in sexual activities, low self-esteem and many more symptoms [1].

$\mathrm{BC}$ is the most common cancer among Arab women and represents between $14 \%$ and $42 \%$ of all female cancers. Moreover, approximately $50 \%$ of the reported cases in the United Arab Emirates (UAE) are women younger than 50 years, compared to only $25 \%$ in other developed countries [2].

In the UAE, BC is a growing health concern, as it is the leading type of cancer among women and ranks second among the most common causes of death. According to the data from the American Institute of Cancer Research, the number of new BC cases in 2018 represented $39.9 \%$ of all cancer types among females in the UAE (Emirati and non-Emirati), with an associated mortality rate of $12.4 \%$. The cumulative risk of BC in the UAE has increased for years, reaching $5.8 \%$ in 2018 [3].

There is a large amount of research on the QoL of women with BC in Western societies, and this research has identified several factors affecting the QoL of women with BC [4-14]. The results have shown that BC survivors report a moderate to high prevalence of psychological morbidities such as depression, anxiety, pain and sleeping disorders. However, research in the Arab world is very limited. Recent reviews [15, 16] studied the QoL of women with $\mathrm{BC}$ in the Middle East (ME) and found that less than one-third of the study participants from the ME had a good QoL.

The few existing studies in the UAE $[4,17-19]$ showed that women in the UAE (Emirati and non-Emirati) have little to poor knowledge about BC. This low level of knowledge is coupled with social, cultural and religious restrictions [18], resulting in low uptake of BC screening services.

However, to our knowledge, no study has assessed QoL among Emirati women with BC. This study will play a leading role in providing information about the QoL of Emirati women with BC that could enable health care policy makers to develop policies that enhance the QoL of women with BC. The study will help in recognizing the aspects of QoL in BC survivorship that lack assets and provide evidence-based data that can help solve problems related to surviving BC for Emirati women.

\section{Methods}

\subsection{Setting and Sampling Method}


A community-based cross-sectional study was conducted from September 2020 to April 2021 among 250 Emirati women. The study participants were recruited from two hospitals in the UAE: Dubai Hospital in the Emirate of Dubai and Tawam Hospital in Al Ain city. Dubai Hospital is the only governmental hospital in Dubai with an oncology center, while Tawam Hospital is considered the cancer referral governmental hospital in the UAE. While Dubai Hospital falls under the Dubai Health Authority, Tawam Hospital falls under the Department of Health of the Emirate of Abu Dhabi, the capital of the UAE.

The lists of patients in both Dubai and Al Ain hospitals were obtained; all registered women were contacted by phone to either administer the survey or determine their interest to take the survey face to face during their next visit. Nonregistered women visiting for consultation or visiting the cancer care unit were also approached to take part in the study through face-to-face interviews.

The list obtained from the Dubai Hospital Cancer Registry had 212 registered women since 2016. Out of these Emirati women, 69 had survived BC, while 13 had not. The list from Tawam Hospital had 113 registered women, 75 registered from 2020 and 38 registered as of August 2021 . The response rate from Dubai Hospital was 100\%, as all 69 Emirati women registered in the hospital took the survey, while the response rate from Tawam Hospital was $66.4 \%$. As the subject was very sensitive, nurses trained by a specialist in BC interviewed the women by explaining the study and asking them all the questions in the questionnaire.

\subsection{Study Instruments}

A structured questionnaire was administered through face-to-face interviews in the hospitals. The questionnaire included three parts. The first part collected information on sociodemographic and reproductive characteristics, including age, level of education, marital status, employment, and smoking habits. It also collected BC data, such as time elapsed since diagnosis, according to which women were defined as having an early diagnosis ( $\leq 1$ year since diagnosis), being in the transitional period ( $>1$ and $\leq 5$ years since diagnosis) and being long-term survivors ( $>5$ to $\leq 10$ years).

The second part assessed the QoL of Emirati women with BC using the European Organization for Research and Treatment of Cancer Quality of Life Cancer-Specific version (EORTC QLQ-C30, v.3.0).

The third part contained the Quality of Life Breast Cancer-Specific version (EORTC QLQ-BR23), which explores five domains: therapy side effects, arm symptoms, breast symptoms, body image, and sexual functioning.

The EORTC QLQ-C30 is a 30-item instrument designed to assess cancer patients' physical, psychological and social functioning [12, 20]. It is composed of nine multi-item scales: five functional scales, a global QoL scale, and three symptom scales (fatigue, pain, and nausea and vomiting). In addition, there are five single-item symptom scales (dyspnea, sleep disturbance, appetite loss, constipation, and diarrhea) and a final item that evaluates the perceived financial impact of the disease.

Each of the first 28 items of this instrument is answered on a scale from 1 (not at all) to 4 (very much). The time frame is the present moment. For item number 29 (on overall general health) and item 30 (on overall QoL), the response options range from 1 (very poor) to 7 (excellent), and the time frame is during the past week.

The QLQ-BR-23 [12, 21] consists of 23 items divided into two multi-item functional scales (body image and sexual functioning), three symptom scales (systemic side effects, breast symptoms, and arm symptoms), and three single-item scales on sexual enjoyment, future perspectives, and upset by hair loss. The response options are on a scale of 1 (not at all) to 4 (very much), and the time frame is during the past week, except for the sexual items (during the past four weeks).

The Arabic versions of the QLQ-C30 and QLQ-BR23 were developed and translated by the EORTC. The validation of the QLQ-C30 and QLQ-BR23 was performed in the Arab population in the UAE in [4].

A professional translator translated the English version of the sociodemographic part into the Arabic language. A second bilingual speaker checked the Arabic version word by word with the English version and then translated it back to English. The content validity of the Arabic version of the questionnaire was assessed by a panel of experts in the field to evaluate the readability, language simplicity and suitability of the items and to evaluate the relationship of each item to the whole scale. The panel was composed of a professor in obstetrics and gynecology, an oncologist, and a public health specialist. Based on their comments, changes were made. The internal consistency reliability of the Arabic version of the questionnaire was assessed using Cronbach's $\alpha$, with coefficients of 0.918 and 0.878 for the EORTC QLQ-C30 and QLQ BR-23, respectively, which suggested relatively good internal consistency.

\subsection{Statistical Analysis}

The collected data were coded, entered, and analyzed using the statistical package SPSS version 26. Descriptive statistics were computed to describe all items of the questionnaire. Scores were calculated for both the EORTC QLQ-C30 and EORTC QLQ-BR23 patient responses using a scoring algorithm recommended by the EORTC $[20,21]$. The scoring algorithm involves first computing the average of the item responses and second transforming the score to a value on a $0-100 \%$ scale.

A high scale score represents a higher response level. Hence, a high score for a functional scale represents a high level of functioning, and a high score for the global health status/QoL represents a high QoL; however, a high score for a symptom scale item corresponds to more frequent and/or more

Page $3 / 21$ 
intense symptoms.

For the functional scales and the global QOL, subjects with problematic functioning were defined as those who scored below $33.3 \%$ (<33.3\%), while subjects in good condition were those who scored $77.7 \%$ or more ( $\geq 66.7 \%$ ). For symptom scales, subjects who scored below $33.3 \%$ were classified as having less severe symptoms, while those scoring $66.7 \%$ or more were classified as having more intense symptoms [22].

One-way analysis of variance (ANOVA) or the independent-sample t test were carried out to test the equality of population means across the categories of each independent variable (predictor) depending on the number of categories for the independent variables. In case the statistical assumptions required for one-way ANOVA and t test were violated, nonparametric tests, namely, Kruskal-Wallis and Mann-Whitney, were used instead. Additional exploration of the differences among the means was determined by post hoc analysis.

Pearson's linear correlation coefficient was computed to assess the linear relationship between each of the outcome variables and each of the quantitative independent variables. Global health status/QoL and the functional and symptom scales served as the dependent variables. The independent variables (age, duration since diagnosis, marital status, educational level, employment status, income, menopausal status, pathological staging, history of metastases, chemotherapy, lumpectomy, mastectomy, lymph node dissection, radiotherapy, and hormonal therapy) were categorized into two categories (yes and no) and served as predictors for the models. $\mathrm{R}^{2}$ was computed, and statistical tests with $\mathrm{p}-\mathrm{values}<0.05$ were considered statistically significant.

\section{Results}

\subsection{Characteristics of the Study Sample}

The mean age of the 250 Emirati women was 53.4 (SD \pm 11.3 ), and the median age was 52 years; the range was 59 ( 27 was the minimum and 86 the maximum). The mean time elapsed since diagnosis was 4.44 (SD \pm 4.3 ), with a minimum of 0 (year 2021) and a maximum of 25 years (year 1996 ) (Table 1) 
Table 1

Demographic data of the participants $(N=250)$

\begin{tabular}{|c|c|c|}
\hline Characteristic & Frequency & Percentage \\
\hline \multicolumn{3}{|l|}{ Age } \\
\hline$\leq 50$ & 107 & 42.8 \\
\hline$>50$ & 143 & 57.2 \\
\hline \multicolumn{3}{|l|}{ Marital Status } \\
\hline Single & 15 & 6.0 \\
\hline Married & 187 & 74.8 \\
\hline Divorced & 16 & 6.4 \\
\hline Widowed & 32 & 12.8 \\
\hline \multicolumn{3}{|l|}{ Education Level } \\
\hline Illiterate & 45 & 18 \\
\hline Primary School & 32 & 12.8 \\
\hline Preparatory School & 23 & 9.2 \\
\hline Secondary School & 56 & 22.4 \\
\hline University Graduate & 94 & 37.6 \\
\hline \multicolumn{3}{|l|}{ Employment } \\
\hline Yes & 58 & 23.2 \\
\hline No & 174 & 69.6 \\
\hline Retired & 18 & 7.2 \\
\hline Menopausal Status & $\mathrm{N}$ & $\%$ \\
\hline Premenopause & 26 & 10.4 \\
\hline Perimenopause & 24 & 9.6 \\
\hline Postmenopause & 153 & 61.2 \\
\hline Menopause due to surgery & 47 & 18.8 \\
\hline \multicolumn{3}{|l|}{ Monthly Income } \\
\hline$<10,000$ AED & 40 & 16.0 \\
\hline 10000-20000 AED & 117 & 46.8 \\
\hline 20000-3-0000 AED & 73 & 29.2 \\
\hline$>30000$ AED & 20 & 8.0 \\
\hline \multicolumn{3}{|l|}{ Time Since Diagnosis } \\
\hline Early Diagnosis & 46 & 18.4 \\
\hline Transitional Period & 136 & 54.4 \\
\hline Long-term Survivors & 68 & 27.2 \\
\hline \multicolumn{3}{|l|}{ Stage } \\
\hline Stage 1 & 67 & 26.8 \\
\hline Stage 2 & 55 & 22.0 \\
\hline Stage 3 & 53 & 21.2 \\
\hline Stage 4 & 12 & 4.8 \\
\hline I don't know & 63 & 25.2 \\
\hline Metastasis & & \\
\hline
\end{tabular}

Page 5/21 


\begin{tabular}{|lll|}
\hline Characteristic & Frequency & Percentage \\
\hline Yes & 29 & 11.6 \\
\hline No & 215 & 86.0 \\
\hline I don't know & 6 & 2.4 \\
\hline Type of Treatment & & \\
\hline Chemotherapy & 181 & $72.4 \%$ \\
\hline Radiotherapy & 146 & $58.4 \%$ \\
\hline Hormonal therapy & 138 & $55.2 \%$ \\
\hline Lumpectomy & 106 & $42.4 \%$ \\
\hline Mastectomy & 128 & $51.2 \%$ \\
\hline Lymph node dissection & 78 & $31.2 \%$ \\
\hline
\end{tabular}

Out of the 250 participants, 99 (39.6\%) were diagnosed in 2020, while 45 (18\%) were diagnosed more than 5 years ago.

The majority of women (70\%) were from Dubai Hospital, while 30\% were from Tawam Hospital. More than half of the participants (56.8\%) were from Dubai, 19.6\% were from Abu-Dhabi, 12\% were from Sharjah, and 11.6\% were from the other emirates (Ajman, Rass al Khaimah, Um Al Quwain, and Fujairah).

The majority of women (95.2\%) never smoked, and $87.6 \%$ of them had children, with a mean number of 4 (SD \pm 3 ) children (a maximum number of 10 children). Approximately $39 \%$ of the participants exercised on a daily basis, and approximately $4 \%$ lived alone. In total, $44 \%$ of them had had a miscarriage, with a mean number of 1 (SD \pm 1.3 ) miscarriages (a maximum number of 6 miscarriages). More than a quarter (25.6\%) of the participants had a family history of $\mathrm{BC}$, and $11.6 \%$ had the cancer spread to other parts of their body.

\subsection{Quality-of-life scale scores}

The global health/QoL mean score of the 250 participants was 74.73 (SD \pm 18.25 ), with a minimum of $16.67 \%$, indicating a good level of wellbeing (Table 2).

On the QLQ-C30 scales, the mean scores for the five functional scales ranged from 68.43 to 82.33 , showing mostly a good level of functional health status. While social functioning scored the highest $(82.33 \pm 28.38)$ among the functional scales, emotional functioning scored the lowest $(68.43 \pm$ 30.02).

On the symptom scales, the most worrying symptom was sleep disturbance $(47.87 \pm 38.46)$, followed by fatigue ( $38.18 \pm 30.31)$ and pain $(29.13 \pm$ 28.01). Financial impact scored the lowest, indicating that most women did not have financial issues related to their cancer. Out of the 250 participants, $5.6-12.4 \%$ had problematic functioning on the functional scales but worse functioning on the symptom scales, as $6.8-45.6 \%$ had problems with symptoms.

On the QLQ-BR23 scales, the range of the mean scores for the functional scales was 50.80 to 80.30 , showing mostly above average to good levels of functional health status. While sexual functioning scored the highest $(86.07 \pm 22.61)$ among the functional scales, future perspective scored the lowest $(50.80 \pm 37.92)$.

On the symptom scales, the most worrying symptom was upset by hair loss ( $61.01 \pm 37.35)$, followed by arm symptoms ( $33.73 \pm 28.08)$. Notably, all symptom scales means had 0 as the minimum score and 100 as the maximum score, with the exception of systemic side effects, for which the maximum was 95.42 . Out of the 250 participants, $2-24 \%$ had problematic functioning on the functional scales but worse functioning on the symptom scales, as $12-40 \%$ had problems. 


\begin{tabular}{|c|c|c|c|c|c|}
\hline Variables & No. of items & Mean (SD) & $95 \% \mathrm{Cl}$ & $\begin{array}{l}\mathrm{N}(\%) \\
(\text { scoring <33.3) }\end{array}$ & $\begin{array}{l}N(\%) \\
\text { (scoring } \geq 66.7)^{b}\end{array}$ \\
\hline \multicolumn{6}{|l|}{ QLQ-C30 } \\
\hline Global health status/QoL & 2 & $74.73(18.25)$ & $72.46-77.00$ & $4(1.6)$ & $190(76.0)$ \\
\hline \multicolumn{6}{|l|}{ Functional scales ${ }^{b}$} \\
\hline Physical functioning & 5 & $69.95(25.13)$ & $66.82-73.08$ & $22(8.8)$ & $166(66.4)$ \\
\hline Role functioning & 2 & $79.13(28.36)$ & $75.60-82.67$ & $14(5.6)$ & $199(79.6)$ \\
\hline Emotional functioning & 4 & $68.43(30.02)$ & $64.69-72.17$ & $31(12.4)$ & $168(67.2)$ \\
\hline Cognitive functioning & 2 & $74.93(27.51)$ & $71.51-78.36$ & $17(6.8)$ & $190(76.0)$ \\
\hline Social functioning & 2 & $82.33(28.38)$ & $78.80-85.87$ & $16(6.4)$ & $209(83.6)$ \\
\hline \multicolumn{6}{|l|}{ Symptom scales ${ }^{c}$} \\
\hline Fatigue & 3 & $38.18(30.31)$ & $34.40-41.95$ & $105(42.0)$ & $58(23.2)$ \\
\hline Nausea and vomiting & 2 & $18.80(27.17)$ & $15.42-22.18$ & $168(67.2)$ & $26(10.4)$ \\
\hline Pain & 2 & $29.13(28.01)$ & $25.64-32.62$ & $128(51.2)$ & $41(16.4)$ \\
\hline Dyspnea & 1 & $20.53(29.66)$ & $16.84-24.23$ & $150(60.0)$ & $39(15.6)$ \\
\hline Sleep disturbance- Insomnia & 1 & $47.87(38.46)$ & $43.08-52.66$ & $70(28.0)$ & $114(45.6)$ \\
\hline Appetite loss & 1 & $25.87(32.96)$ & $21.76-29.97$ & $130(52.0)$ & $48(19.2)$ \\
\hline Constipation & 1 & $26.93(35.27)$ & $22.54-31.33$ & $136(54.4)$ & $56(22.4)$ \\
\hline Diarrhea & 1 & $12.13(26.54)$ & $8.83-15.44$ & $195(78.0)$ & $22(8.8)$ \\
\hline Financial impact & 1 & $9.2(23.13)$ & $6.32-12.08$ & $207(82.8)$ & $17(6.8)$ \\
\hline \multicolumn{6}{|l|}{ QLQ-BR23 } \\
\hline \multicolumn{6}{|l|}{ Functional scales ${ }^{b}$} \\
\hline Body image & 4 & $80.30(25.73)$ & $77.09-83.51$ & $17(6.8)$ & $204(81.6)$ \\
\hline Sexual functioning & 2 & $86.07(22.61)$ & $83.25-88.88$ & $5(2.0)$ & $224(89.6)$ \\
\hline Sexual enjoyment $(N=78)$ & 1 & $63.68(30.48)$ & $56.80-70.53$ & $6(2.4)$ & $54(21.6)$ \\
\hline Future perspective & 1 & $50.80(37.92)$ & $46.08-55.52$ & $60(24.0)$ & $121(48.4)$ \\
\hline \multicolumn{6}{|l|}{ Symptom scales ${ }^{c}$} \\
\hline Systemic side effect & 7 & $31.98(25.37)$ & $28.82-35.14$ & $134(53.6)$ & $31(12.4)$ \\
\hline Breast symptoms & 4 & $26.93(27.90)$ & $23.46-30.41$ & $154(61.6)$ & $33(13.2)$ \\
\hline Arm symptoms & 3 & $33.73(28.08)$ & $30.23-37.23$ & $117(46.8)$ & $48(19.2)$ \\
\hline Upset by hair loss & 1 & $61.01(37.35)$ & $55.16-66.86$ & $28(11.2)$ & $100(40.0)$ \\
\hline \multicolumn{6}{|c|}{$\begin{array}{l}\text { aFor the functional scales, women scoring }<33.3 \% \text { had problems; those scoring } \geq 66.7 \% \text { had good functioning. For the symptom } \\
\text { scales/symptoms, women scoring }<33.3 \% \text { had good functioning; those scoring } \geq 66.7 \% \text { had problems. }\end{array}$} \\
\hline \multicolumn{6}{|c|}{${ }^{\mathrm{b}}$ For the functional scales, higher scores indicate better functioning. } \\
\hline
\end{tabular}

\subsection{Factors Associated with Quality-of-life Scale Scores}

\subsubsection{Global Health, Functional, and Emotional Scales on the QLQ-C30}


Table 3 shows that there were significant differences in the global health/QoL means across categories of monthly income $(P=0.018)$, physical activity $(P=0.0004)$, history of metastases $(P=0.001)$, and type of treatment $(P=0.045$ for chemotherapy). Post hoc analysis showed that participants who had regular physical activity, had high income, had no history of metastases, and were not treated with chemotherapy seemed to have better global health-related QoL. 
Table 3

Global health and functional scales on the QLQ-C30 by independent variables $(N=250)^{a}$

\begin{tabular}{|c|c|c|c|c|c|c|}
\hline \multirow[t]{3}{*}{ Characteristic } & \multirow{3}{*}{$\begin{array}{l}\text { Global health } \\
\text { Status/Qol } \\
\text { Mean (SD) }\end{array}$} & \multicolumn{5}{|c|}{ Functional scales in QLQ-C30b } \\
\hline & & $\begin{array}{l}\text { Physical } \\
\text { functioning }\end{array}$ & $\begin{array}{l}\text { Role functioning } \\
\text { Mean (SD) }\end{array}$ & $\begin{array}{l}\text { Emotional } \\
\text { functioning }\end{array}$ & $\begin{array}{l}\text { Cognitive } \\
\text { functioning }\end{array}$ & $\begin{array}{l}\text { Social } \\
\text { functioning }\end{array}$ \\
\hline & & Mean (SD) & & Mean (SD) & Mean (SD) & Mean (SD) \\
\hline \multicolumn{7}{|l|}{ Age } \\
\hline$\leq 50$ years & 74.84 (18.06) & 70.65 (24.84) & 77.88 (30.18) & 58.26 (31.02) & 69.47 (27.99) & $72.43(30.47)$ \\
\hline$>50$ years & 74.65 (18.45) & $69.42(25.42)$ & 80.07 (26.99) & 76.05 (26.94) & $79.02(26.52)$ & $89.74(24.30)$ \\
\hline$P$ value & 0.969 & 0.725 & 0.638 & $<0.0001$ & 0.002 & $<0.0001$ \\
\hline \multicolumn{7}{|l|}{$\begin{array}{l}\text { Time since } \\
\text { diagnosis }\end{array}$} \\
\hline Early Diagnosis & $75.00(18.00)$ & 63.79 (27.78) & 69.20 (32.39) & $65.03(28.74)$ & 73.91 (28.47) & 77.90 (26.07) \\
\hline Transitional & 75.92 (18.14) & $71.23(25.03)$ & $81.62(26.86)$ & $65.01(30.19)$ & 74.02 (27.57) & $81.99(28.84)$ \\
\hline \multicolumn{7}{|l|}{ Period } \\
\hline Long-term & 72.18 (18.64) & 71.57 (23.11) & $80.88(27.36)$ & 77.57 (29.02) & 77.45 (26.98) & $86.03(28.88)$ \\
\hline \multicolumn{7}{|l|}{ Survivors } \\
\hline$P$ value & 0.417 & 0.257 & 0.043 & 0.002 & 0.637 & 0.037 \\
\hline \multicolumn{7}{|l|}{ Marital Status } \\
\hline Single & 75.00 (19.16) & 72.89 (25.38) & 87.78 (22.24) & $51.11(34.63)$ & 65.56 (37.52) & 76.67 (28.73) \\
\hline Married & 74.20 (17.86) & 70.05 (24.38) & $79.23(28.47)$ & $67.20(29.88)$ & 75.94 (26.27) & $81.11(29.37)$ \\
\hline Divorced & $82.81(16.52)$ & $72.50(25.40)$ & $82.29(29.48)$ & 66.67 (31.18) & 72.92 (30.35) & 83.33 (29.81) \\
\hline Widowed & $73.70(20.64)$ & 66.67 (29.77) & 72.91 (29.56) & 84.63 (21.07) & 74.48 (28.39) & $91.67(19.40)$ \\
\hline$P$ value & 0.350 & 0.894 & 0.279 & 0.001 & 0.817 & 0.108 \\
\hline \multicolumn{7}{|l|}{ Education Level } \\
\hline Illiterate & 73.33 (18.00) & 63.85 (28.37) & 72.59 (29.34) & $79.26(26.45)$ & $78.89(26.45)$ & $92.96(18.63)$ \\
\hline Primary School & 75.26 (17.77) & $74.79(20.02)$ & $88.02(24.40)$ & 78.91 (27.92) & 83.85 (26.26) & 91.67 (21.59) \\
\hline Preparatory School & 73.91 (23.74) & $65.51(24.10)$ & $78.26(24.33)$ & 77.90 (26.78) & $77.54(22.81)$ & 92.75 (19.35) \\
\hline Secondary School & $71.43(17.61)$ & $67.50(25.11)$ & $72.92(31.87)$ & 63.54 (34.69) & $70.24(27.84)$ & $73.21(32.52)$ \\
\hline University Graduate & 77.39 (17.35) & $73.76(24.84)$ & $83.16(26.83$ & $60.28(27.26)$ & $72.16(28.75)$ & $76.95(30.54)$ \\
\hline$P$-value & 0.355 & 0.130 & 0.012 & $<0.0001$ & 0.057 & $<0.0001$ \\
\hline \multicolumn{7}{|l|}{ Employment } \\
\hline Yes & 75.86 (16.72) & $74.37(24.24)$ & $84.20(25.44)$ & $60.63(26.66)$ & $75.29(25.60)$ & 76.15 (29.64) \\
\hline No & 73.75 (18.32) & $67.93(25.44)$ & 77.78 (28.01) & $70.88(30.83)$ & $74.62(27.74)$ & 84.39 (27.65) \\
\hline Retired & 80.56 (21.77) & 75.19 (23.63) & $75.93(38.87)$ & 69.91 (29.59) & 76.85 (32.41) & $82.41(29.96)$ \\
\hline$P$ value & 0.132 & 0.113 & 0.234 & 0.014 & 0.819 & 0.028 \\
\hline \multicolumn{7}{|l|}{ Monthly Income } \\
\hline$<10,000$ AED & $69.58(21.89)$ & $64.33(24.56)$ & $72.08(29.81)$ & $61.46(32.78)$ & $69.58(33.52)$ & $75.42(33.97)$ \\
\hline 10000-20000 AED & 72.93 (17.91) & $68.26(24.11)$ & 79.06 (30.02) & $70.51(29.04)$ & 74.93 (25.49) & 83.05 (27.42) \\
\hline 20000-3-0000 AED & 78.65 (15.96) & $77.44(23.87)$ & $80.82(26.45)$ & $72.26(30.71)$ & $79.00(26.21)$ & $89.73(22.33)$ \\
\hline > 30000 AED & 81.25 (16.64) & 63.67 (31.45) & $87.50(19.40)$ & 56.25 (23.24) & $70.83(30.05)$ & $65.00(32.84)$ \\
\hline
\end{tabular}




\begin{tabular}{|c|c|c|c|c|c|c|}
\hline \multirow[t]{3}{*}{ Characteristic } & \multirow{3}{*}{$\begin{array}{l}\text { Global health } \\
\text { Status/Qol } \\
\text { Mean (SD) }\end{array}$} & \multicolumn{5}{|c|}{ Functional scales in QLQ-C30 } \\
\hline & & $\begin{array}{l}\text { Physical } \\
\text { functioning }\end{array}$ & $\begin{array}{l}\text { Role functioning } \\
\text { Mean (SD) }\end{array}$ & $\begin{array}{l}\text { Emotional } \\
\text { functioning }\end{array}$ & $\begin{array}{l}\text { Cognitive } \\
\text { functioning }\end{array}$ & $\begin{array}{l}\text { Social } \\
\text { functioning }\end{array}$ \\
\hline & & Mean (SD) & & Mean (SD) & Mean (SD) & Mean (SD) \\
\hline$P$ value & 0.018 & 0.007 & 0.232 & 0.018 & 0.399 & $<0.001$ \\
\hline \multicolumn{7}{|l|}{ Physical Activity } \\
\hline Yes & $79.08(17.76)$ & $77.42(22.52)$ & $85.88(21.05)$ & $66.67(29.48)$ & $77.38(25.13)$ & $75.85(32.12)$ \\
\hline No & $71.93(18.06)$ & $65.13(25.61)$ & $74.78(31.52)$ & $69.57(30.41)$ & $73.36(29.92)$ & $88.51(24.92)$ \\
\hline$P$ value & 0.004 & $<0.0001$ & 0.013 & 0.331 & 0.382 & 0.003 \\
\hline \multicolumn{7}{|l|}{ Menopausal Status } \\
\hline Premenopause & $71.47(20.97)$ & $69.74(20.76)$ & $75.64(30.63)$ & $59.94(29.54)$ & $73.72(29.50)$ & $72.44(31.25)$ \\
\hline Perimenopause & $78.13(20.09)$ & $62.50(35.16)$ & $68.06(38.04)$ & $72.92(27.39)$ & $75.69(25.05)$ & $72.22(32.10)$ \\
\hline Postmenopause & $74.40(17.73)$ & $70.59(24.94)$ & $82.68(25.64)$ & $73.37(28.92)$ & $78.10(26.18)$ & $88.67(24.97)$ \\
\hline $\begin{array}{l}\text { Menopause due to } \\
\text { Surgery }\end{array}$ & $75.89(17.57)$ & $71.77(21.86)$ & $75.18(28.63)$ & $54.79(30.73)$ & $64.89(30.14)$ & $72.34(30.15)$ \\
\hline$P$ value & 0.555 & 0.883 & 0.111 & $<0.0001$ & 0.042 & $<0.0001$ \\
\hline \multicolumn{7}{|l|}{ Children } \\
\hline Yes & 74.16(18.20) & 69.89(24.64) & 78.16(28.71) & 69.18(29.73) & 75.57(26.96) & $82.42(28.54)$ \\
\hline No & 78.76(18.36) & 70.32(28.79) & $86.02(25.13)$ & 63.17(32.04) & 70.43(31.24) & 81.72(27.67) \\
\hline$P$ value & 0.227 & 0.618 & 0.091 & 0.271 & 0.398 & 0.688 \\
\hline \multicolumn{7}{|l|}{ Stage } \\
\hline Stage 1 & $78.48(16.10)$ & 77.21 (22.24) & 86.57 (23.97) & $66.04(29.49)$ & $79.10(24.85)$ & $86.82(25.71)$ \\
\hline Stage 2 & 73.03 (17.27) & $65.33(23.36)$ & $78.48(28.09)$ & $65.15(28.42)$ & $69.70(26.66)$ & $72.73(33.39)$ \\
\hline Stage 3 & 70.28 (18.89) & 70.31 (21.35) & $80.82(24.55)$ & $64.62(31.90)$ & $69.50(26.90)$ & $77.36(27.56)$ \\
\hline Stage 4 & $73.61(21.57)$ & $67.22(31.33)$ & 75.00 (35.18) & $66.67(30.77)$ & 73.61 (33.68) & $86.11(30.01)$ \\
\hline$P$ value & 0.069 & 0.025 & 0.168 & 0.981 & 0.093 & 0.023 \\
\hline \multicolumn{7}{|l|}{ Metastases } \\
\hline Yes & $64.66(17.49)$ & $62.76(22.50)$ & 77.59 (27.92) & $72.13(31.44)$ & 76.44 (29.72) & $84.48(29.19)$ \\
\hline No & $76.36(17.70)$ & $71.53(25.046)$ & $79.77(28.33)$ & $68.06(29.83)$ & 75.04 (27.10) & $82.56(28.09)$ \\
\hline$P$ value & 0.001 & 0.027 & 0.599 & 0.347 & 0.657 & 0.430 \\
\hline \multicolumn{7}{|l|}{ Chemotherapy } \\
\hline Yes & $73.30(19.02)$ & $68.69(24.72)$ & 79.37 (27.97) & $66.16(30.97)$ & $71.92(27.97)$ & 79.37 (30.09) \\
\hline No & 78.50 (15.55) & 73.24 (26.06) & 78.50 (29.58) & $74.40(26.67)$ & 82.85 (24.75) & $90.10(21.64)$ \\
\hline$P$ value & 0.045 & 0.110 & 0.929 & 0.069 & 0.002 & 0.006 \\
\hline \multicolumn{7}{|l|}{ Radiotherapy } \\
\hline Yes & $74.03(18.30)$ & 70.37 (22.95) & $80.37(26.67)$ & $67.12(28.88)$ & $73.86(28.22)$ & $78.77(30.23)$ \\
\hline No & 75.72 (18.22) & $69.36(28.02)$ & $77.40(30.63)$ & 70.27 (31.60) & $76.44(26.54)$ & $87.34(24.85)$ \\
\hline$P$ value & 0.438 & 0.701 & 0.759 & 0.190 & 0.563 & 0.006 \\
\hline \multicolumn{7}{|l|}{ Lumpectomy } \\
\hline Yes & $73.90(19.69)$ & $68.49(26.26)$ & $80.19(26.64)$ & $68.40(28.26)$ & $73.90(27.12)$ & 78.14 (31.32) \\
\hline
\end{tabular}




\begin{tabular}{|c|c|c|c|c|c|c|}
\hline \multirow[t]{3}{*}{ Characteristic } & \multirow{3}{*}{$\begin{array}{l}\text { Global health } \\
\text { Status/Qol } \\
\text { Mean (SD) }\end{array}$} & \multicolumn{5}{|c|}{ Functional scales in QLQ-C30 } \\
\hline & & $\begin{array}{l}\text { Physical } \\
\text { functioning }\end{array}$ & $\begin{array}{l}\text { Role functioning } \\
\text { Mean (SD) }\end{array}$ & $\begin{array}{l}\text { Emotional } \\
\text { functioning }\end{array}$ & $\begin{array}{l}\text { Cognitive } \\
\text { functioning }\end{array}$ & $\begin{array}{l}\text { Social } \\
\text { functioning }\end{array}$ \\
\hline & & Mean (SD) & & Mean (SD) & Mean (SD) & Mean (SD) \\
\hline No & 75.35 (17.15) & $71.02(24.30)$ & $78.36(29.64)$ & $68.46(31.35)$ & $75.69(27.87)$ & $85.42(25.69)$ \\
\hline$P$ value & 0.504 & 0.564 & 0.935 & 0.605 & 0.536 & 0.030 \\
\hline \multicolumn{7}{|l|}{ Mastectomy } \\
\hline Yes & $75.00(17.56)$ & $70.42(23.42)$ & $79.43(28.56)$ & $66.21(31.41)$ & 75.78 (26.61) & $83.46(26.08)$ \\
\hline No & 74.45 (19.01) & $69.45(26.90)$ & $78.83(28.27)$ & $70.77(28.43)$ & 74.04 (28.51) & $81.15(30.68)$ \\
\hline$P$ value & 0.659 & 0.801 & 0.606 & 0.360 & 0.828 & 0.792 \\
\hline \multicolumn{7}{|l|}{$\begin{array}{l}\text { Lymph Node } \\
\text { Dissection }\end{array}$} \\
\hline Yes & 72.76 (18.59) & $68.72(23.40)$ & $82.69(27.84)$ & $57.69(31.66)$ & $66.03(30.08)$ & $69.87(32.68)$ \\
\hline No & $75.63(18.07)$ & $70.50(25.93)$ & $77.52(28.53)$ & $73.30(28.01)$ & $78.97(25.34)$ & $87.98(24.27)$ \\
\hline$P$ value & 0.246 & 0.350 & 0.090 & $<0.0001$ & 0.001 & $<0.0001$ \\
\hline \multicolumn{7}{|c|}{ 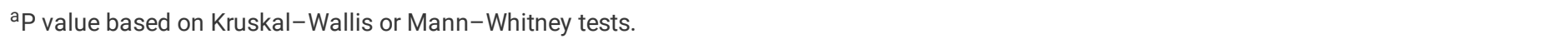 } \\
\hline${ }^{\mathrm{b}}$ For the functic & higher score & e better func & & & & \\
\hline
\end{tabular}

Furthermore, significant differences in the physical functioning means were observed across categories of monthly income $(P=0.007)$, physical activity $(P<0.0001)$, history of metastases $(P=0.027)$, and disease stage $(P=0.025)$. Post hoc analysis showed that participants who had regular physical activity, had high income, had no history of metastases, and were in early pathological staging had better functioning on the physical functioning scale.

Differences in the emotional functioning means were observed across categories of age, educational level, menopausal status, lymph node dissection $(P<0.0001$ each), time since diagnosis $(P=0.002)$, history of metastases $(P=0.001)$, employment status $(P=0.014)$, and monthly income $(P=0.018$. Post hoc analysis showed that participants who were aged above 50, were long-term survivors, were not employed, went through normal menopause, and had no history of lymph node dissection had better emotional functioning (Table 3).

\subsubsection{Symptom Scales on the QLQ-C30}

With the exception of appetite loss and constipation, there were significant differences in all symptom scales across age categories. Women aged below 50 had worse symptoms on the symptom scales than those aged above 50. Moreover, there were significant differences in the mean pain scores by time since diagnosis $(P=0.026)$, pathological staging $(P=0.007)$, menopausal status $(P=0.010)$, education level $(P=0.012)$, monthly income $(P=$ $0.005)$ and metastasis categories $(P=0.035)$. Post hoc analysis revealed that those who were older, were long-term survivors, were postmenopausal, and had a minimum education level (primary) experienced more pain.

Furthermore, there were significant differences in financial impact across age, menopausal status, monthly income, lymph node dissection, and chemotherapy. Post hoc analysis revealed that those who were older, postmenopausal, had an average salary, and had undergone lymph node dissection or chemotherapy experienced more financial impact.

\subsubsection{Functional and Symptom Scales on the QLQ-BR 23}

As shown in Table 4, differences in the means of body images were significant among the categories of all independent variables with the exception of a history of metastases, radiology, and lumpectomy. Post hoc analysis showed that those who were younger, were employed, were premenopausal, were single, had low income, had undergone chemotherapy or dissection and were highly educated seemed to have poorer body image. 
Table 4

Functional and symptom scales on the QLQ-BR23 by independent variables $(N=250)^{\text {a }}$

\begin{tabular}{|c|c|c|c|c|c|c|c|c|}
\hline \multirow{3}{*}{ Characteristic } & \multicolumn{4}{|c|}{ Functional scales in BR $23^{b}$} & \multicolumn{4}{|c|}{ Symptom scale in BR $23^{c}$} \\
\hline & Body image & $\begin{array}{l}\text { Sexual } \\
\text { functioning }\end{array}$ & $\begin{array}{l}\text { Sexual } \\
\text { enjoyment }\end{array}$ & $\begin{array}{l}\text { Future } \\
\text { perspective }\end{array}$ & $\begin{array}{l}\text { Systemic- } \\
\text { therapy side }\end{array}$ & $\begin{array}{l}\text { Breast } \\
\text { symptoms }\end{array}$ & $\begin{array}{l}\text { Arm } \\
\text { symptoms }\end{array}$ & $\begin{array}{l}\text { Upset by } \\
\text { hair loss }\end{array}$ \\
\hline & & Mean (SD) & Mean (SD) & Mean (SD) & Mean (SD) & Mean (SD) & Mean (SD) & Mean (SD) \\
\hline \multicolumn{9}{|l|}{ Age } \\
\hline$\leq 50$ years & $\begin{array}{l}72.98 \\
(27.99)\end{array}$ & $\begin{array}{l}74.92 \\
(26.93)\end{array}$ & $\begin{array}{l}58.18 \\
(30.91)\end{array}$ & $\begin{array}{l}42.68 \\
(37.98)\end{array}$ & $\begin{array}{l}38.63 \\
(26.00)\end{array}$ & $\begin{array}{l}34.11 \\
(27.29)\end{array}$ & $\begin{array}{l}39.56 \\
(26.00)\end{array}$ & $\begin{array}{l}67.57 \\
(34.88)\end{array}$ \\
\hline$>50$ years & $\begin{array}{l}85.78 \\
(22.48)\end{array}$ & $\begin{array}{l}94.41 \\
(13.84)\end{array}$ & $\begin{array}{l}76.81 \\
(25.49)\end{array}$ & $\begin{array}{l}56.88 \\
(36.86)\end{array}$ & $\begin{array}{l}27.01 \\
(23.79)\end{array}$ & $\begin{array}{l}21.56 \\
(27.29)\end{array}$ & $\begin{array}{l}29.37 \\
(28.87)\end{array}$ & $\begin{array}{l}55.29 \\
(38.68)\end{array}$ \\
\hline$P$ value & $<0.0001$ & $<0.0001$ & 0.015 & 0.003 & $<0.0001$ & $<0.0001$ & 0.001 & 0.047 \\
\hline \multicolumn{9}{|l|}{$\begin{array}{l}\text { Time Since } \\
\text { Diagnosis }\end{array}$} \\
\hline Early Diagnosis & $\begin{array}{l}76.45 \\
(25.36)\end{array}$ & $\begin{array}{l}83.33 \\
(22.77)\end{array}$ & $\begin{array}{l}69.70 \\
(27.04)\end{array}$ & $\begin{array}{l}46.38 \\
(40.05)\end{array}$ & $\begin{array}{l}39.03 \\
(28.17)\end{array}$ & $\begin{array}{l}36.59 \\
(32.23)\end{array}$ & $\begin{array}{l}39.86 \\
(27.17)\end{array}$ & $\begin{array}{l}67.68 \\
(32.79)\end{array}$ \\
\hline $\begin{array}{l}\text { Transitional } \\
\text { Period }\end{array}$ & $\begin{array}{l}80.27 \\
(24.70)\end{array}$ & $\begin{array}{l}82.84 \\
(25.25)\end{array}$ & $\begin{array}{l}59.57 \\
(32.55)\end{array}$ & $\begin{array}{l}48.53 \\
(37.81)\end{array}$ & $\begin{array}{l}31.30 \\
(25.27)\end{array}$ & $\begin{array}{l}27.51 \\
(26.69)\end{array}$ & $\begin{array}{l}33.74 \\
(27.30)\end{array}$ & $\begin{array}{l}57.92 \\
(39.24)\end{array}$ \\
\hline $\begin{array}{l}\text { Long-term } \\
\text { Survivors }\end{array}$ & $\begin{array}{l}82.97 \\
(27.97)\end{array}$ & $\begin{array}{l}94.36 \\
(13.07)\end{array}$ & $\begin{array}{l}70.37 \\
(26.06)\end{array}$ & $\begin{array}{l}58.33 \\
(36.14)\end{array}$ & $\begin{array}{l}28.57 \\
(22.93)\end{array}$ & $\begin{array}{l}19.24 \\
(25.20)\end{array}$ & $\begin{array}{l}29.58 \\
(29.84)\end{array}$ & $\begin{array}{l}61.59 \\
(37.16)\end{array}$ \\
\hline$P$ value & 0.070 & 0.001 & 0.339 & 0.147 & 0.151 & 0.004 & 0.092 & 0.526 \\
\hline \multicolumn{9}{|l|}{ Marital Status } \\
\hline Single & $\begin{array}{l}75.00 \\
(29.55)\end{array}$ & $\begin{array}{l}94.44 \\
(10.29)\end{array}$ & - & $\begin{array}{l}31.11 \\
(29.46)\end{array}$ & $\begin{array}{l}37.46 \\
(26.38)\end{array}$ & $\begin{array}{l}38.89 \\
(25.33)\end{array}$ & $\begin{array}{l}37.78 \\
(26.49)\end{array}$ & $\begin{array}{l}43.59 \\
(34.39)\end{array}$ \\
\hline Married & $\begin{array}{l}78.39 \\
(27.02)\end{array}$ & $\begin{array}{l}82.35 \\
(24.70)\end{array}$ & $\begin{array}{l}62.16 \\
(30.38)\end{array}$ & $\begin{array}{l}50.45 \\
(38.70)\end{array}$ & $\begin{array}{l}32.31 \\
(24.93)\end{array}$ & $\begin{array}{l}26.43 \\
(27.04)\end{array}$ & $\begin{array}{l}34.11 \\
(27.48)\end{array}$ & $\begin{array}{l}61.98 \\
(37.09)\end{array}$ \\
\hline Divorced & $\begin{array}{l}82.81 \\
(19.60)\end{array}$ & $95.83(9.62)$ & $\begin{array}{l}88.89 \\
(19.25)\end{array}$ & $\begin{array}{l}60.42 \\
(38.91)\end{array}$ & $\begin{array}{l}27.98 \\
(30.56)\end{array}$ & $\begin{array}{l}27.60 \\
(31.87)\end{array}$ & $\begin{array}{l}34.72 \\
(31.66)\end{array}$ & $\begin{array}{l}83.33 \\
(27.89)\end{array}$ \\
\hline Widowed & $\begin{array}{l}92.70 \\
(12.66)\end{array}$ & $98.96(4.10)$ & $100(-)$ & $\begin{array}{l}57.29 \\
(34.11)\end{array}$ & $\begin{array}{l}29.46 \\
(25.38)\end{array}$ & $\begin{array}{l}23.96 \\
(31.66)\end{array}$ & $\begin{array}{l}29.17 \\
(31.14)\end{array}$ & $\begin{array}{l}59.65 \\
(40.94)\end{array}$ \\
\hline$P$ value & 0.029 & $<0.0001$ & 0.131 & 0.113 & 0.546 & 0.165 & 0.576 & 0.146 \\
\hline \multicolumn{9}{|l|}{ Education Level } \\
\hline Illiterate & $\begin{array}{l}91.48 \\
(14.38)\end{array}$ & $\begin{array}{l}95.93 \\
(10.75)\end{array}$ & $66.67(0.00)$ & $\begin{array}{l}66.67 \\
(32.57)\end{array}$ & $\begin{array}{l}23.17 \\
(20.97)\end{array}$ & $\begin{array}{l}20.74 \\
(24.98)\end{array}$ & $\begin{array}{l}31.11 \\
(29.64)\end{array}$ & $\begin{array}{l}56.52 \\
(36.84)\end{array}$ \\
\hline Primary School & $\begin{array}{l}84.11 \\
(25.95)\end{array}$ & $\begin{array}{l}93.75 \\
(22.70)\end{array}$ & $\begin{array}{l}22.22 \\
(38.49)\end{array}$ & $\begin{array}{l}61.46 \\
(33.98)\end{array}$ & $\begin{array}{l}26.04 \\
(26.14)\end{array}$ & $\begin{array}{l}16.93 \\
(22.05)\end{array}$ & $\begin{array}{l}29.17 \\
(24.07)\end{array}$ & $\begin{array}{l}62.50 \\
(43.67)\end{array}$ \\
\hline $\begin{array}{l}\text { Preparatory } \\
\text { School }\end{array}$ & $\begin{array}{l}85.87 \\
(14.31)\end{array}$ & $\begin{array}{l}82.61 \\
(29.08)\end{array}$ & $\begin{array}{l}74.07 \\
(43.39)\end{array}$ & $\begin{array}{l}57.97 \\
(40.47)\end{array}$ & $\begin{array}{l}33.95 \\
(27.10)\end{array}$ & $\begin{array}{l}28.26 \\
(28.95)\end{array}$ & $\begin{array}{l}31.88 \\
(23.52)\end{array}$ & $\begin{array}{l}50.98 \\
(42.68)\end{array}$ \\
\hline $\begin{array}{l}\text { Secondary } \\
\text { School }\end{array}$ & $\begin{array}{l}80.06 \\
(28.32)\end{array}$ & $\begin{array}{l}83.93 \\
(24.20)\end{array}$ & $\begin{array}{l}55.56 \\
(24.34)\end{array}$ & $\begin{array}{l}45.83 \\
(38.44)\end{array}$ & $\begin{array}{l}31.97 \\
(22.97)\end{array}$ & $\begin{array}{l}28.57 \\
(29.81)\end{array}$ & $\begin{array}{l}32.54 \\
(27.94)\end{array}$ & $\begin{array}{l}62.63 \\
(39.75)\end{array}$ \\
\hline $\begin{array}{l}\text { University } \\
\text { Graduate }\end{array}$ & $\begin{array}{l}72.43 \\
(28.13)\end{array}$ & $\begin{array}{l}80.85 \\
(22.26)\end{array}$ & $\begin{array}{l}68.22 \\
(28.13)\end{array}$ & $\begin{array}{l}40.78 \\
(37.60)\end{array}$ & $\begin{array}{l}37.74 \\
(26.79)\end{array}$ & $\begin{array}{l}32.00 \\
(28.67)\end{array}$ & $\begin{array}{l}37.71 \\
(29.70)\end{array}$ & $\begin{array}{l}63.81 \\
(33.93)\end{array}$ \\
\hline$P$-value & $<0.0001$ & $<0.0001$ & 0.070 & 0.001 & 0.020 & 0.037 & 0.560 & 0.758 \\
\hline \multicolumn{9}{|l|}{ Employment } \\
\hline Yes & $\begin{array}{l}70.55 \\
(29.79)\end{array}$ & $\begin{array}{l}73.85 \\
(21.88)\end{array}$ & $\begin{array}{l}65.74 \\
(29.26)\end{array}$ & $\begin{array}{l}39.66 \\
(38.21)\end{array}$ & $\begin{array}{l}37.44 \\
(30.11)\end{array}$ & $\begin{array}{l}38.51 \\
(32.25)\end{array}$ & $\begin{array}{l}38.51 \\
(28.63)\end{array}$ & $\begin{array}{l}61.11 \\
(32.02)\end{array}$ \\
\hline \multicolumn{9}{|c|}{ aP value based on Kruskal-Wallis or Mann-Whitney tests. } \\
\hline \multicolumn{9}{|c|}{${ }^{b}$ For the functional scales, higher scores indicate better functioning. } \\
\hline \multicolumn{9}{|c|}{${ }^{\mathrm{C}}$ For the symptom scales, higher scores indicate worse functioning. } \\
\hline $1 \$=3.67 \mathrm{AED}$ & & & & & & & & \\
\hline
\end{tabular}




\begin{tabular}{|c|c|c|c|c|c|c|c|c|}
\hline \multirow[b]{2}{*}{ No } & \multicolumn{4}{|c|}{ Functional scales in BR $23^{b}$} & \multicolumn{4}{|c|}{ Symptom scale in BR $23^{c}$} \\
\hline & $\begin{array}{l}84.05 \\
(23.41)\end{array}$ & $\begin{array}{l}90.42 \\
(21.45)\end{array}$ & $\begin{array}{l}56.86 \\
(31.28)\end{array}$ & $\begin{array}{l}56.13 \\
(37.04)\end{array}$ & $\begin{array}{l}30.43 \\
(23.71)\end{array}$ & $\begin{array}{l}24.28 \\
(26.24)\end{array}$ & $\begin{array}{l}32.06 \\
(28.12)\end{array}$ & $\begin{array}{l}61.68 \\
(39.60)\end{array}$ \\
\hline Retired & $\begin{array}{l}75.46 \\
(25.48)\end{array}$ & $\begin{array}{l}83.33 \\
(22.14)\end{array}$ & $\begin{array}{l}83.33 \\
(25.20)\end{array}$ & $\begin{array}{l}35.19 \\
(35.19)\end{array}$ & $\begin{array}{l}29.37 \\
(23.11)\end{array}$ & $\begin{array}{l}15.28 \\
(15.19)\end{array}$ & $\begin{array}{l}34.57 \\
(25.53)\end{array}$ & $\begin{array}{l}53.33 \\
(35.83)\end{array}$ \\
\hline$P$ value & 0.001 & $<0.0001$ & 0.079 & 0.003 & 0.404 & 0.005 & 0.277 & 0.683 \\
\hline \multicolumn{9}{|l|}{ Monthly Income } \\
\hline$<10,000$ AED & $\begin{array}{l}68.75 \\
(28.42)\end{array}$ & $\begin{array}{l}77.50 \\
(32.81)\end{array}$ & $\begin{array}{l}35.71 \\
(30.56)\end{array}$ & $\begin{array}{l}35.00 \\
(39.19)\end{array}$ & $\begin{array}{l}42.62 \\
(27.34)\end{array}$ & $\begin{array}{l}42.92 \\
(32.93)\end{array}$ & $\begin{array}{l}45.56 \\
(32.88)\end{array}$ & $\begin{array}{l}66.67 \\
(40.57)\end{array}$ \\
\hline $\begin{array}{l}10000-20000 \\
\text { AED }\end{array}$ & $\begin{array}{l}81.20 \\
(26.94)\end{array}$ & $\begin{array}{l}87.04 \\
(20.31)\end{array}$ & $\begin{array}{l}63.89 \\
(26.87)\end{array}$ & $\begin{array}{l}54.99 \\
(37.73)\end{array}$ & $\begin{array}{l}29.30 \\
(23.98)\end{array}$ & $\begin{array}{l}23.01 \\
(24.99)\end{array}$ & $\begin{array}{l}34.76 \\
(25.79)\end{array}$ & $\begin{array}{l}54.79 \\
(37.83)\end{array}$ \\
\hline $\begin{array}{l}20000-3-0000 \\
\text { AED }\end{array}$ & $\begin{array}{l}84.93 \\
(21.41)\end{array}$ & $\begin{array}{l}91.10 \\
(17.59)\end{array}$ & $\begin{array}{l}72.55 \\
(24.25)\end{array}$ & $\begin{array}{l}52.97 \\
(35.50)\end{array}$ & $\begin{array}{l}26.29 \\
(22.28)\end{array}$ & $\begin{array}{l}18.84 \\
(21.56)\end{array}$ & $\begin{array}{l}20.24 \\
(22.09)\end{array}$ & $\begin{array}{l}66.67 \\
(34.16)\end{array}$ \\
\hline > 30000 AED & $\begin{array}{l}81.25 \\
(21.94)\end{array}$ & $\begin{array}{l}79.17 \\
(22.21)\end{array}$ & $\begin{array}{l}84.85 \\
(27.34)\end{array}$ & $\begin{array}{l}50.00 \\
(39.74)\end{array}$ & $\begin{array}{l}47.14 \\
(29.35)\end{array}$ & $\begin{array}{l}47.50 \\
(32.68)\end{array}$ & $\begin{array}{l}53.33 \\
(28.29)\end{array}$ & $\begin{array}{l}64.71 \\
(36.27)\end{array}$ \\
\hline$P$ value & 0.011 & 0.021 & 0.001 & 0.032 & 0.001 & $<0.0001$ & $<0.0001$ & 0.267 \\
\hline \multicolumn{9}{|l|}{$\begin{array}{l}\text { Physical } \\
\text { Activity }\end{array}$} \\
\hline Yes & $\begin{array}{l}74.32 \\
(29.13)\end{array}$ & $\begin{array}{l}82.48 \\
(23.86)\end{array}$ & $\begin{array}{l}65.74 \\
(34.26)\end{array}$ & $\begin{array}{l}48.30 \\
(38.03)\end{array}$ & $\begin{array}{l}32.75 \\
(28.15)\end{array}$ & $\begin{array}{l}29.51 \\
(27.66)\end{array}$ & $\begin{array}{l}36.28 \\
(28.39)\end{array}$ & $\begin{array}{l}64.33 \\
(38.25)\end{array}$ \\
\hline No & $\begin{array}{l}84.16 \\
(22.55)\end{array}$ & $\begin{array}{l}88.38 \\
(21.53)\end{array}$ & $\begin{array}{l}61.90 \\
(27.12)\end{array}$ & $\begin{array}{l}52.41 \\
(37.90)\end{array}$ & $\begin{array}{l}31.49 \\
(23.49)\end{array}$ & $\begin{array}{l}25.27 \\
(28.02)\end{array}$ & $\begin{array}{l}32.09 \\
(27.86)\end{array}$ & $\begin{array}{l}59.15 \\
(36.91)\end{array}$ \\
\hline$P$ value & 0.005 & 0.014 & 0.469 & 0.406 & 0.945 & 0.149 & 0.219 & 0.345 \\
\hline \multicolumn{9}{|l|}{$\begin{array}{l}\text { Menopausal } \\
\text { Status }\end{array}$} \\
\hline Premenopause & $\begin{array}{l}70.51 \\
(28.99)\end{array}$ & $\begin{array}{l}69.23 \\
(35.49)\end{array}$ & $\begin{array}{l}45.24 \\
(36.06)\end{array}$ & $\begin{array}{l}34.62 \\
(38.27)\end{array}$ & $\begin{array}{l}37.91 \\
(23.15)\end{array}$ & $\begin{array}{l}41.03 \\
(23.80)\end{array}$ & $\begin{array}{l}38.89 \\
(22.93)\end{array}$ & $\begin{array}{l}61.90 \\
(38.42)\end{array}$ \\
\hline Perimenopause & $\begin{array}{l}81.25 \\
(23.47)\end{array}$ & $\begin{array}{l}79.17 \\
(27.03)\end{array}$ & $\begin{array}{l}57.58 \\
(26.21)\end{array}$ & $\begin{array}{l}59.72 \\
(36.75)\end{array}$ & $\begin{array}{l}34.13 \\
(30.07)\end{array}$ & $\begin{array}{l}30.21 \\
(30.97)\end{array}$ & $\begin{array}{l}40.74 \\
(34.31)\end{array}$ & $\begin{array}{l}69.05 \\
(38.04)\end{array}$ \\
\hline Postmenopause & $\begin{array}{l}84.20 \\
(24.22)\end{array}$ & $\begin{array}{l}92.37 \\
(15.65)\end{array}$ & $\begin{array}{l}72.22 \\
(27.80)\end{array}$ & $\begin{array}{l}56.64 \\
(37.48)\end{array}$ & $\begin{array}{l}28.73 \\
(24.83)\end{array}$ & $\begin{array}{l}21.79 \\
(26.22)\end{array}$ & $\begin{array}{l}29.77 \\
(27.59)\end{array}$ & $\begin{array}{l}58.42 \\
(38.59)\end{array}$ \\
\hline $\begin{array}{l}\text { Menopause due } \\
\text { to Surgery }\end{array}$ & $\begin{array}{l}72.52 \\
(27.30)\end{array}$ & $\begin{array}{l}78.37 \\
(23.03)\end{array}$ & $\begin{array}{l}66.67 \\
(28.43)\end{array}$ & $\begin{array}{l}36.17 \\
(33.93)\end{array}$ & $\begin{array}{l}38.20 \\
(24.63)\end{array}$ & $\begin{array}{l}34.22 \\
(29.91)\end{array}$ & $\begin{array}{l}40.19 \\
(27.27)\end{array}$ & $\begin{array}{l}64.52 \\
(33.26)\end{array}$ \\
\hline$P$ value & 0.001 & $<0.0001$ & 0.076 & 0.001 & 0.046 & $<0.0001$ & 0.034 & 0.724 \\
\hline \multicolumn{9}{|l|}{ Children } \\
\hline Yes & $80.48(25.90)$ & $85.31(23.32)$ & 63.01(29.69) & $51.14(38.35)$ & $31.72(24.72)$ & $26.75(27.89)$ & $33.59(28.37)$ & 63.02(37.20) \\
\hline No & 79.03(24.90) & $91.40(16.03)$ & 73.33(43.46) & $48.39(35.32)$ & $33.79(29.95)$ & $28.23(28.36)$ & $34.77(26.41)$ & $48.48(36.70)$ \\
\hline$P$ value & 0.583 & 0.264 & 0.312 & 0.723 & 0.919 & 0.900 & 0.676 & 0.082 \\
\hline \multicolumn{9}{|l|}{ Stage } \\
\hline Stage 1 & $\begin{array}{l}83.33 \\
(22.89)\end{array}$ & $\begin{array}{l}83.58 \\
(24.36)\end{array}$ & $\begin{array}{l}59.09 \\
(28.97)\end{array}$ & $\begin{array}{l}53.23 \\
(38.95)\end{array}$ & $\begin{array}{l}23.67 \\
(23.45)\end{array}$ & $\begin{array}{l}24.01 \\
(26.21)\end{array}$ & $\begin{array}{l}28.36 \\
(25.17)\end{array}$ & $\begin{array}{l}50.51 \\
(39.19)\end{array}$ \\
\hline Stage 2 & $\begin{array}{l}76.67 \\
(22.36)\end{array}$ & $\begin{array}{l}83.94 \\
(25.45)\end{array}$ & $\begin{array}{l}63.16 \\
(31.22)\end{array}$ & $\begin{array}{l}42.42 \\
(36.55)\end{array}$ & $\begin{array}{l}43.46 \\
(22.09)\end{array}$ & $\begin{array}{l}30.30 \\
(27.38)\end{array}$ & $\begin{array}{l}38.99 \\
(28.84)\end{array}$ & $\begin{array}{l}71.32 \\
(25.80)\end{array}$ \\
\hline Stage 3 & $\begin{array}{l}69.50 \\
(33.45)\end{array}$ & $\begin{array}{l}79.56 \\
(25.87)\end{array}$ & $\begin{array}{l}58.67 \\
(32.32)\end{array}$ & $\begin{array}{l}49.06 \\
(38.46)\end{array}$ & $\begin{array}{l}39.08 \\
(28.76)\end{array}$ & $\begin{array}{l}34.12 \\
(30.10)\end{array}$ & $\begin{array}{l}40.46 \\
(31.13)\end{array}$ & $\begin{array}{l}63.06 \\
(39.11)\end{array}$ \\
\hline
\end{tabular}

ap value based on Kruskal-Wallis or Mann-Whitney tests.

${ }^{b}$ For the functional scales, higher scores indicate better functioning.

${ }^{\mathrm{C}}$ For the symptom scales, higher scores indicate worse functioning.

$1 \$=3.67 \mathrm{AED}$ 
Functional scales in BR $23^{\text {b }}$

\begin{tabular}{|c|c|c|c|c|c|c|c|c|}
\hline Stage 4 & $\begin{array}{l}88.19 \\
(13.97)\end{array}$ & $\begin{array}{l}90.28 \\
(13.22)\end{array}$ & $\begin{array}{l}80.00 \\
(18.26)\end{array}$ & $\begin{array}{l}38.89 \\
(39.78)\end{array}$ & $\begin{array}{l}36.90 \\
(27.78)\end{array}$ & $\begin{array}{l}41.67 \\
(37.61)\end{array}$ & $\begin{array}{l}38.89 \\
(27.83)\end{array}$ & $\begin{array}{l}70.00 \\
(33.15)\end{array}$ \\
\hline$P$ value & 0.044 & 0.571 & 0.524 & 0.369 & $<0.0001$ & 0.179 & 0.085 & 0.144 \\
\hline \multicolumn{9}{|c|}{ Metastases } \\
\hline Yes & $\begin{array}{l}81.61 \\
(27.85)\end{array}$ & $\begin{array}{l}85.63 \\
(20.76)\end{array}$ & $\begin{array}{l}53.33 \\
(28.11)\end{array}$ & $\begin{array}{l}39.08 \\
(36.81)\end{array}$ & $\begin{array}{l}40.56 \\
(23.69)\end{array}$ & $\begin{array}{l}34.20 \\
(31.52)\end{array}$ & $\begin{array}{l}41.38 \\
(31.97)\end{array}$ & $\begin{array}{l}66.67 \\
(33.33)\end{array}$ \\
\hline No & $\begin{array}{l}80.00 \\
(25.69)\end{array}$ & $\begin{array}{l}85.81 \\
(23.10)\end{array}$ & $\begin{array}{l}65.20 \\
(30.71)\end{array}$ & $\begin{array}{l}52.25 \\
(37.91)\end{array}$ & $\begin{array}{l}30.32 \\
(25.16)\end{array}$ & $\begin{array}{l}25.16 \\
(26.58)\end{array}$ & $\begin{array}{l}32.04 \\
(26.90)\end{array}$ & $\begin{array}{l}59.38 \\
(38.32)\end{array}$ \\
\hline$P$ value & 0.452 & 0.767 & 0.219 & 0.079 & 0.028 & 0.186 & 0.125 & 0.417 \\
\hline \multicolumn{9}{|c|}{ Chemotherapy } \\
\hline Yes & $\begin{array}{l}77.44 \\
(27.91)\end{array}$ & $\begin{array}{l}85.36 \\
(22.61)\end{array}$ & $\begin{array}{l}66.12 \\
(30.73)\end{array}$ & $\begin{array}{l}48.43 \\
(36.76)\end{array}$ & $\begin{array}{l}35.12 \\
(26.17)\end{array}$ & $\begin{array}{l}25.92 \\
(26.44)\end{array}$ & $\begin{array}{l}36.65 \\
(28.01)\end{array}$ & $\begin{array}{l}62.27 \\
(38.27)\end{array}$ \\
\hline No & $\begin{array}{l}87.80 \\
(16.82)\end{array}$ & $\begin{array}{l}87.92 \\
(22.67)\end{array}$ & $\begin{array}{l}54.90 \\
(28.73)\end{array}$ & $\begin{array}{l}57.00 \\
(40.46)\end{array}$ & $\begin{array}{l}23.74 \\
(21.19)\end{array}$ & $\begin{array}{l}29.59 \\
(31.46)\end{array}$ & $\begin{array}{l}26.09 \\
(27.00)\end{array}$ & $\begin{array}{l}55.56 \\
(33.14)\end{array}$ \\
\hline$P$ value & 0.031 & 0.196 & 0.130 & 0.116 & 0.002 & 0.678 & 0.004 & 0.261 \\
\hline \multicolumn{9}{|c|}{ Radiotherapy } \\
\hline Yes & $\begin{array}{l}76.66 \\
(27.81)\end{array}$ & $\begin{array}{l}85.16 \\
(22.16)\end{array}$ & $\begin{array}{l}71.70 \\
(30.24)\end{array}$ & $\begin{array}{l}46.12 \\
(36.99)\end{array}$ & $\begin{array}{l}35.84 \\
(26.23)\end{array}$ & $\begin{array}{l}29.85 \\
(28.12)\end{array}$ & $\begin{array}{l}39.35 \\
(27.50)\end{array}$ & $\begin{array}{l}62.96 \\
(36.23)\end{array}$ \\
\hline No & $\begin{array}{l}85.42 \\
(21.59)\end{array}$ & $\begin{array}{l}87.34 \\
(23.28)\end{array}$ & $\begin{array}{l}46.67 \\
(23.57)\end{array}$ & $\begin{array}{l}57.37 \\
(38.43)\end{array}$ & $\begin{array}{l}26.56 \\
(23.16)\end{array}$ & $\begin{array}{l}22.84 \\
(27.18)\end{array}$ & $\begin{array}{l}25.85 \\
(27.10)\end{array}$ & $\begin{array}{l}57.78 \\
(39.23)\end{array}$ \\
\hline$P$ value & 0.009 & 0.198 & $<0.0001$ & 0.021 & 0.006 & 0.026 & $<0.0001$ & 0.456 \\
\hline \multicolumn{9}{|c|}{ Lumpectomy } \\
\hline Yes & $\begin{array}{l}79.40 \\
(24.81)\end{array}$ & $\begin{array}{l}86.48 \\
(21.96)\end{array}$ & $\begin{array}{l}64.58 \\
(31.61)\end{array}$ & $\begin{array}{l}51.57 \\
(37.97)\end{array}$ & $\begin{array}{l}33.74 \\
(26.63)\end{array}$ & $\begin{array}{l}30.82 \\
(28.73)\end{array}$ & $\begin{array}{l}34.80 \\
(27.90)\end{array}$ & $\begin{array}{l}62.63 \\
(34.85)\end{array}$ \\
\hline No & $\begin{array}{l}80.96 \\
(26.46)\end{array}$ & $\begin{array}{l}85.76 \\
(23.15)\end{array}$ & $\begin{array}{l}63.04 \\
(30.00)\end{array}$ & $\begin{array}{l}50.23 \\
(38.02)\end{array}$ & $\begin{array}{l}30.69 \\
(24.42)\end{array}$ & $\begin{array}{l}24.07 \\
(27.01)\end{array}$ & $\begin{array}{l}32.95 \\
(28.29)\end{array}$ & $\begin{array}{l}59.86 \\
(39.18)\end{array}$ \\
\hline$P$ value & 0.339 & 0.892 & 0.752 & 0.784 & 0.444 & 0.035 & 0.578 & 0.797 \\
\hline \multicolumn{9}{|c|}{ Mastectomy } \\
\hline Yes & $\begin{array}{l}76.37 \\
(28.23)\end{array}$ & $\begin{array}{l}85.03 \\
(24.20)\end{array}$ & $\begin{array}{l}59.52 \\
(31.70)\end{array}$ & $\begin{array}{l}46.35 \\
(37.47)\end{array}$ & $\begin{array}{l}31.92 \\
(24.63)\end{array}$ & $\begin{array}{l}25.65 \\
(26.96)\end{array}$ & $\begin{array}{l}37.33 \\
(27.98)\end{array}$ & $\begin{array}{l}63.41 \\
(37.99)\end{array}$ \\
\hline No & $\begin{array}{l}84.43 \\
(22.19)\end{array}$ & $\begin{array}{l}87.16 \\
(20.86)\end{array}$ & $\begin{array}{l}68.52 \\
(28.66)\end{array}$ & $\begin{array}{l}55.46 \\
(38.00)\end{array}$ & $\begin{array}{l}32.05 \\
(26.23)\end{array}$ & $\begin{array}{l}28.28 \\
(28.90)\end{array}$ & $\begin{array}{l}29.96 \\
(27.81)\end{array}$ & $\begin{array}{l}58.44 \\
(36.74)\end{array}$ \\
\hline$P$ value & 0.024 & 0.675 & 0.197 & 0.058 & 0.888 & 0.577 & 0.028 & 0.338 \\
\hline \multicolumn{9}{|c|}{$\begin{array}{l}\text { Lymph Node } \\
\text { Dissection }\end{array}$} \\
\hline Yes & $\begin{array}{l}71.37 \\
(30.26)\end{array}$ & $\begin{array}{l}83.76 \\
(24.02)\end{array}$ & $\begin{array}{l}67.86 \\
(33.31)\end{array}$ & $\begin{array}{l}47.01 \\
(37.00)\end{array}$ & $\begin{array}{l}42.37 \\
(25.27)\end{array}$ & $\begin{array}{l}32.37 \\
(27.88)\end{array}$ & $\begin{array}{l}42.02 \\
(28.41)\end{array}$ & $\begin{array}{l}64.94 \\
(35.55)\end{array}$ \\
\hline No & $\begin{array}{l}84.35 \\
(22.34)\end{array}$ & $\begin{array}{l}87.11 \\
(21.93)\end{array}$ & $\begin{array}{l}61.33 \\
(28.86)\end{array}$ & $\begin{array}{l}52.52 \\
(38.32)\end{array}$ & $\begin{array}{l}27.27 \\
(24.05)\end{array}$ & $\begin{array}{l}24.47 \\
(27.63)\end{array}$ & $\begin{array}{l}29.97 \\
(27.19)\end{array}$ & $\begin{array}{l}58.75 \\
(38.34)\end{array}$ \\
\hline$P$ value & $<0.0001$ & 0.218 & 0.256 & 0.296 & $<0.0001$ & 0.017 & 0.002 & 0.358 \\
\hline
\end{tabular}

${ }^{\text {ap }}$ value based on Kruskal-Wallis or Mann-Whitney tests.

${ }^{b}$ For the functional scales, higher scores indicate better functioning.

CFor the symptom scales, higher scores indicate worse functioning.

$1 \$=3.67$ AED

Symptom scale in BR $23^{\mathrm{c}}$

34.20

41.38

66.67

59.38

(38.32)

0.417

80.00
$(25.69)$

77.44

87.80

0.031

76.66

85.42

(21.59)

(23.28)

(23.57)

(38.43)

(23.16)

(27.18)

(27.10)

(39.23)

Lumpectomy

Yes

Better sexual functioning was observed for women who were aged above $50(P<0.0001)$, were long-term survivors $(P=0.001)$, were not working $(P<$ $0.0001)$ and had gone through natural menopause $(P<0.0001)$. Furthermore, post hoc analysis showed that women aged above 50 and those who 
had radiology as treatment tend to have better sexual enjoyment functioning.

More intense upset by hair loss was noted among women who were aged above $50(P=0.047)$.

Additionally, women aged above 50 had worse systemic side effects $(P<0.0001)$ and breast $(P<0.001)$ and arm $(P=0.001)$ symptoms.

Women who had surgical menopause complained of more severe systemic side effects $(P=0.017)$ and breast $(P=0.006)$ and arm $(P=0.019)$ symptoms than women who had menopause naturally.

Women who were recently diagnosed complained of more severe arm symptoms $(P=0.001)$, and women in advanced pathological staging complained of more severe systemic side effects.

\subsubsection{Predictors of Quality of Life}

Table 5 below summarizes the adjusted regression models for the QLQ-C30. 
Table 5

Linear regression model with parameter estimates for the QLQ functional scales

\begin{tabular}{|c|c|c|c|c|c|c|c|c|c|c|c|c|}
\hline \multirow[t]{2}{*}{ Variable } & \multicolumn{2}{|c|}{$\begin{array}{l}\text { Global Health/QoL } \\
\text { score }\end{array}$} & \multicolumn{2}{|c|}{$\begin{array}{l}\text { Physical } \\
\text { functioning }\end{array}$} & \multicolumn{2}{|c|}{ Role functioning } & \multicolumn{2}{|c|}{$\begin{array}{l}\text { Emotional } \\
\text { functioning }\end{array}$} & \multicolumn{2}{|c|}{$\begin{array}{l}\text { Cognitive } \\
\text { functioning }\end{array}$} & \multicolumn{2}{|c|}{$\begin{array}{l}\text { Social } \\
\text { functioning }\end{array}$} \\
\hline & $\beta$ & $P$ & $\beta$ & $\mathbf{P}$ & $\beta$ & $\mathbf{P}$ & $\boldsymbol{\beta}$ & $\mathbf{P}$ & $\beta$ & $\mathbf{P}$ & $\beta$ & $\mathbf{P}$ \\
\hline Constant & 80.704 & $<0.0001$ & 74.274 & $<.0001$ & 73.346 & $<.0001$ & 65.078 & $\dot{0} .0001$ & 66.378 & $<.0001$ & 87.544 & $<0.0001$ \\
\hline Age $>50$ & -.079 & .412 & -.147 & .134 & .050 & .615 & .262 & .004 & .115 & .219 & .074 & .419 \\
\hline \multicolumn{13}{|l|}{$\mathrm{No}=0$} \\
\hline \multicolumn{13}{|l|}{ Yes $=1$} \\
\hline Married & -.063 & .409 & .026 & .732 & .025 & .753 & .047 & .510 & .124 & .093 & .018 & .808 \\
\hline \multicolumn{13}{|l|}{$\mathrm{No}=0$} \\
\hline \multicolumn{13}{|l|}{ Yes $=1$} \\
\hline Education & -.003 & .972 & .033 & .708 & -.079 & .383 & -.220 & .008 & -.125 & .142 & -.197 & .019 \\
\hline \multicolumn{13}{|l|}{$\mathrm{No}=0$} \\
\hline \multicolumn{13}{|l|}{ Yes $=1$} \\
\hline Employment & .008 & .921 & .013 & .873 & .138 & .099 & .021 & .776 & .152 & .051 & -.002 & .979 \\
\hline \multicolumn{13}{|l|}{$\mathrm{No}=0$} \\
\hline \multicolumn{13}{|l|}{ Yes $=1$} \\
\hline High income & .242 & .002 & .127 & .099 & .160 & .043 & .031 & .660 & .062 & .403 & .059 & .416 \\
\hline \multicolumn{13}{|l|}{$\mathrm{No}=0$} \\
\hline \multicolumn{13}{|l|}{ Yes $=1$} \\
\hline Menopause & .042 & .619 & .100 & .244 & .113 & .199 & -.077 & .336 & -.022 & .789 & .098 & .227 \\
\hline \multicolumn{13}{|l|}{$\mathrm{No}=0$} \\
\hline \multicolumn{13}{|l|}{ Yes = 1} \\
\hline Advanced stage & -.052 & .510 & 024 & .766 & -.039 & .635 & .034 & 649 & -.084 & .276 & .027 & .721 \\
\hline \multicolumn{13}{|l|}{$\mathrm{No}=0$} \\
\hline \multicolumn{13}{|l|}{ Yes $=1$} \\
\hline $\begin{array}{l}\text { Long-time } \\
\text { survivors }\end{array}$ & -.033 & .677 & .070 & .381 & .044 & .588 & .074 & .316 & .071 & .352 & -.038 & .613 \\
\hline \multicolumn{13}{|l|}{$\mathrm{No}=0$} \\
\hline \multicolumn{13}{|l|}{ Yes $=1$} \\
\hline Metastases & -.140 & .079 & -.141 & .081 & -.013 & .874 & .046 & .537 & .168 & .031 & .083 & .272 \\
\hline $\mathrm{No}=0$ & & & & & & & & & & & & \\
\hline Yes $=1$ & & & & & & & & & & & & \\
\hline Chemotherapy & -.170 & .035 & -.164 & .047 & -.021 & .799 & -.035 & .648 & -.150 & .056 & -.151 & .051 \\
\hline $\mathrm{No}=0$ & & & & & & & & & & & & \\
\hline Yes $=1$ & & & & & & & & & & & & \\
\hline Radiotherapy & -.021 & .805 & -.034 & .696 & -.087 & .323 & .049 & .542 & .064 & .436 & -.104 & .199 \\
\hline $\mathrm{No}=0$ & & & & & & & & & & & & \\
\hline Yes $=1$ & & & & & & & & & & & & \\
\hline Lumpectomy & .064 & .550 & .043 & .689 & .121 & .272 & .113 & .259 & .150 & .149 & .097 & .339 \\
\hline$\beta$ : Standardized & efficient & & & & & & & & & & & \\
\hline P: Significance & & & & & & & & & & & & \\
\hline
\end{tabular}




\begin{tabular}{|c|c|c|c|c|c|c|c|c|c|c|c|c|}
\hline \multirow[t]{2}{*}{ Variable } & \multicolumn{2}{|c|}{$\begin{array}{l}\text { Global Health/QoL } \\
\text { score }\end{array}$} & \multicolumn{2}{|c|}{$\begin{array}{l}\text { Physical } \\
\text { functioning }\end{array}$} & \multicolumn{2}{|c|}{ Role functioning } & \multicolumn{2}{|c|}{$\begin{array}{l}\text { Emotional } \\
\text { functioning }\end{array}$} & \multicolumn{2}{|c|}{$\begin{array}{l}\text { Cognitive } \\
\text { functioning }\end{array}$} & \multicolumn{2}{|c|}{$\begin{array}{l}\text { Social } \\
\text { functioning }\end{array}$} \\
\hline & $\beta$ & $\mathbf{P}$ & $\beta$ & $\mathbf{P}$ & $\beta$ & $\mathbf{P}$ & $\beta$ & $\mathbf{P}$ & $\beta$ & $\mathbf{P}$ & $\beta$ & $\mathbf{P}$ \\
\hline \multicolumn{13}{|l|}{ No $=0$} \\
\hline \multicolumn{13}{|l|}{ Yes $=1$} \\
\hline Mastectomy & .028 & .781 & .022 & .833 & .071 & .501 & .034 & .723 & .194 & .050 & .157 & .107 \\
\hline \multicolumn{13}{|l|}{ No $=0$} \\
\hline \multicolumn{13}{|l|}{ Yes $=1$} \\
\hline $\begin{array}{l}\text { Lymph node } \\
\text { dissection }\end{array}$ & -.063 & .469 & -.174 & .050 & .038 & .674 & -.179 & .029 & -.203 & .017 & -.184 & .027 \\
\hline \multicolumn{13}{|l|}{$\mathrm{No}=0$} \\
\hline \multicolumn{13}{|l|}{ Yes $=1$} \\
\hline $\begin{array}{l}\text { Hormonal } \\
\text { therapy }\end{array}$ & -.030 & .709 & .040 & .631 & -.098 & .244 & -.013 & .861 & -.052 & .507 & -.050 & .517 \\
\hline \multicolumn{13}{|l|}{ No $=0$} \\
\hline \multicolumn{13}{|l|}{ Yes $=1$} \\
\hline$R^{2}$ & 0.140 & & 0.112 & & 0.076 & & 0.238 & & 0.192 & & 0.217 & \\
\hline$P$ Value & 0.036 & & 0.148 & & 0.546 & & $<0.0001$ & & 0.001 & & $<0.0001$ & \\
\hline \multicolumn{13}{|c|}{ B: Standardized Coefficient Beta } \\
\hline P: Significanc & & & & & & & & & & & & \\
\hline
\end{tabular}

As shown in Table 5, the predictors explained $14 \%$ of the variation in global health, $23.8 \%$ of the variation in emotional functioning, $19.2 \%$ of the variation in cognitive functioning, and $21.7 \%$ of the variation in social functioning. Monthly income was the only predictor that had a significant effect on global health/QoL given the other predictors in the model $(P=0.002)$. While age was significant only in emotional functioning $(P=0.004)$, education was a significant predictor in the emotional and social functioning models $(P=0.008$ and 0.019 , respectively). Metastases and mastectomy were significant only in the cognitive functioning model $(P=0.008$ and 0.019 , respectively). Lymph node dissection was significant in the emotional, cognitive, and social functioning models $(P=0.029,0.017$, and 0.027 , respectively).

The important results of the regression analysis of the QLQ-C30 symptoms scales are that predictors explained $17.5 \%$ of fatigue, $16.3 \%$ of pain, and $23.4 \%$ of insomnia. Additionally, radiology was the only significant predictor in explaining fatigue, education was more important in explaining pain ( $P$ $=0.035$ ), and advanced staging was important in explaining insomnia (Table of all results can be found in the Appendix).

From the linear regression model with parameter estimates for the QLQ-BR23 functional and symptom scales (Appendix), the predictors explained between $16.2 \%$ and $46.5 \%$ of variation in all scales of the QLQ-BR23 with the exception of the upset by hair loss symptom. Late survivor was the only predictor that had a significant effect on breast symptoms given the other predictors in the model $(P=0.009)$, while radiology was the only predictor that had a significant effect on arm symptoms $(P=0.006)$.

Age and employment were significant predictors of sexual functioning $(P=<0.0001$ and 0.018 , respectively). High income and mastectomy were significant predictors in the sexual enjoyment model $(P=0.020$ and 0.044 , respectively).

\section{Discussion}

This study is one of the few studies exploring the QoL of Emirati women with BC, and it revealed that Emirati women survivors of BC have good QoL and functioning but worse symptom experience. The most worrying symptoms for Emirati women were sleep disturbance, fatigue, and pain. A history of metastases and chemotherapy had a major effect across the domains of QoL in Emirati women.

Similarly, on the specific disease tool QLQ-BR23, the Emirati women seemed to perform above average to very good on the functional domains and poorly on the symptom scales. The most worrying symptoms were upset by hair loss and arm symptoms.

\subsection{Comparison with Previous Literature}


The mean global health/QoL of Emirati women with BC was higher (74.73) than that of other women in the Gulf Corporate Council (GCC). For example, the mean global health score was 63.9 in Bahrain [23], 64 and 67.45 in the KSA [24, 25], and 45.3 in Kuwait [26]. This variability could be attributed to different study populations, different tools used to measure the outcome, different sampling techniques and different times elapsed since diagnosis.

Emirati women seemed to perform well on the five functional scales, with means ranging from 68.43 to 82.33 , showing mostly a good level of functional health status. Social functioning scored the highest among Emirati women, which was similar to Bahraini, Saudi, and Kuwaiti women. Emotional functioning scored the lowest among Emirati women, which was similar to Bahraini women [23], but different from Kuwaiti and Saudi women, who scored lowest in physical functioning $[25,26]$. Emirati women had good functioning, with only $5.6-12.4 \%$ having problematic functioning on the functional scales, which was similar to Kuwaiti women (5.8-11.8\%) [26] but better than Bahraini women (3.8-21.8\%) [23].

Emirati women did worse on the symptom scales, as they scored higher on all symptom scales except financial impact. Indeed, while 1.7-17\% of Bahraini women [23] were found to have had bad symptom experience, this figure was 8.8-45.6\% for Emirati women.

Regarding financial impact, Emirati women were much better off than other Arab women in the region, with a score of 9.2, which is much lower than the scores of Bahraini women (34.58), Kuwaiti women (31.2), and Saudi women (17.13). This could be attributed to the fact that healthcare in the UAE is known to be among some of the finest in the world, offering a high standard of medical care in state-of-the art facilities. Healthcare facilities are run by the Dubai Health Authority (DHA), which oversees both public and private healthcare and replaces the Department of Health and Medical Services [27]. Health insurance is mandatory for all nationals and UAE residents. For UAE nationals, public hospitals and clinics are low-cost to free.

The most worrying symptom for Emirati women was sleep disturbance, followed by fatigue and pain, which was similar to studies on women in Bahrain, Kuwait and Saudi Arabia [23, 25, 26].

In accordance with the results from the QoL general scale, on the disease-specific scales (QLQ-BR23), Emirati women were above average to very good on the functional scales and poor on the symptom scales, as $12.4-40 \%$ had bad experience with BC symptoms, which was slightly similar to Kuwaiti women (6.7-40.8\%), [26] but worse than Bahraini women (1.7-14.2\%) [23] and Saudi women (9.5-26.8\%) [25]

Among functional scales, sexual functioning scored the highest among Emirati women, which indicates better functioning. This was similar to Kuwaiti women but contrary to Bahraini and Saudi women, who scored the lowest. This finding should be interpreted with caution, as sexual functioning and enjoyment were perceived and approached differently in various studies due to the sensitivity of the topic and the conservative nature of the community. This scale showed the lowest reliability in a study investigating the reliability and validity of the Arabic versions of the EORTC QLQ-C30 and QLQ-BR23 questionnaires and had to be removed from the reliability analysis because of the very low coefficient values [28].

For Emirati women with BC, the most worrying symptom was upset by hair loss, followed by arm symptoms, which was similar to all other Arab women in the region [23-26, 29,30]. These topics should be given special care and attention. Physiotherapy and hair care options should be discussed and included in the comprehensive rehabilitation care provided to patients.

\subsection{Factors Associated with Quality-of-Life Scores}

The results of the study indicated associations between global health/QoL and monthly income, physical activity, history of metastases, and chemotherapy. Emirati women who had regular physical activity, had high income, had no history of metastases, and were not treated with chemotherapy seemed to have better global health-related QoL. Age was not associated with global health/QoL, which was similar to what was found in Bahraini [23], Kuwaiti [26], and Saudi women [25].

Women who were younger, were employed, were premenopausal, were single, had low income, had undergone chemotherapy or dissection and were highly educated seemed to have poorer body image. Therefore, although doing better on the physical side, younger women did worse on the emotional and body image scales. This is in line with the literature illustrating body image and sexuality issues as disturbing potential consequences of treatment for younger women with BC in particular [31]. Furthermore, monthly income was the only predictor that had a significant effect on global health/QoL given the other predictors in the regression model.

Our study indicated that Emirati women with BC experience problems in some of the QOL domains, and further research in this direction is recommended, especially in the emotional domain, to understand how better this domain could be evaluated and managed among Emirati women with special consideration of cultural sensitivity.

The study highlighted important aspects of the disease, such as side effects of the treatment and the need to explain them to patients along with ways of coping and adjustment.

Furthermore, we recommend that special attention be given to women with metastatic history, as the impact on their QoL is substantial.

This study has several strengths, such as the random sampling method, the use of validated tools to measure the outcome, and the use of a standardized score for analysis; most importantly, it is one of the few studies that included Emirati women with BC as participants. This study also has few limitations, such as that the generalization of the results is limited to patients attending the two hospitals of the study population and that the response rate among the two study locations was variable.

Page $18 / 21$ 


\section{Conclusions}

Emirati women were shown to have a good QoL compared to women in the region, and they performed well on the functional scales but poorly on the symptom scales. While social functioning scored the highest, emotional functioning scored the lowest. Factors associated with a decline in the domains of QoL included age, income, education, metastases, mastectomy, and lymph node dissection. The study contributes to a better understanding of the QoL of Emirati women with BC and the different factors that affect their wellbeing, which could lead to enhanced care and support for Emirati women with BC.

\section{List Of Abbreviations}

QoL; Quality of Life

BC: Breast Cancer

EORTC QLQ-C30: European Organization for Research and Treatment of Cancer Quality of Life Cancer Specific Version

EORTC QLQ-BR23: European Organization for Research and Treatment of Cancer Quality of Life Breast Cancer-Specific version

\section{Declarations}

\section{Ethics Approval and Consent to Participate}

Ethical clearance was obtained from the Zayed University Research and Ethics Committee [ZU20_013_F], Dubai Health Authority Medical Research Committee [DSREC-01/2020_22\}, and Abu Dhabi Health Research and Technology Ethics Committee [DOH/CVDC/2021/327]. Institutional review boards at participating institutions approved procedures and protocols. All procedures performed in this study were in accordance with the ethical standards of the institutional and national research committees and with the 1964 Helsinki declaration and its later amendments or comparable ethical standards. All data sampling and collection methods were performed in accordance with the relevant guidelines and regulations provided by the above mentioned institutions.

The Emirati women on the hospital lists were invited to participate in the study on a voluntary basis. The study purpose was explained to the patients, and informed consent was obtained from participants and from legal guardians of illiterate participants. Consent was obtained for all forms of personally identifiable data including biomedical, clinical, and biometric data. However, confidentiality and privacy were maintained, and any identifiable data was numerically coded so that it could not be traced back to the participant. No organs or tissues were obtained for this study.

\section{Consent for Publication}

Not applicable

\section{Availability of Data and Materials}

The datasets analyzed during the current study are available from the corresponding author on reasonable request.

\section{Competing Interests}

The authors declare that they have no competing interests.

\section{Funding}

Grant Support: RIF Grant R19074, Research Office, Zayed University

\section{Authors' Contributions}

LS participated in the design of the study, performed data collection and analysis, and drafted the manuscript. GJ participated in the design of the study, advised on the results section, completed the discussion section, and revised the manuscript. SK participated in the design of the study and revised the manuscript. ST helped in obtaining approval and data collection from $\mathrm{DH}$, and MA helped in obtaining approval and data collection from TH. All authors read and approved the final manuscript.

\section{Acknowledgments}

The questionnaires were used with authorization from the EORTC Quality of Life Study Group. Additionally, we would like to thank all participants, physicians and nurses in the Oncology Centre in Dubai and Tawam hospitals for their great help and support during this study.

\section{References}


1. Wild CP, Weiderpass E, Stewart BW. World cancer report: cancer research for cancer prevention. Lyon, France: International Agency for Research on Cancer; 2020.

2. El Saghir NS, Khalil MK, Eid T, El Kinge AR, Charafeddine M, Geara F, et al. Trends in epidemiology and management of breast cancer in developing Arab countries: a literature and registry analysis. Int J Surg. 2007;5:225-33.

3. World Health Organization. International Agency for Research on Cancer. 2020. https://www.iarc.fr/. Accessed Jul $20,2020$.

4. Awad MA, Denic S, El Taji H. Validation of the European Organization for Research and Treatment of Cancer quality of life questionnaires for Arabic-speaking populations. Ann N Y Acad Sci. 2008;1138:146-54.

5. Scott NW, Fayers PM, Aaronson NK, Bottomley A, de Graeff A, Groenvold M, et al. The relationship between overall quality of life and its subdimensions was influenced by culture: analysis of an international database. J Clin Epidemiol. 2008;61:788-95.

6. Diener E. Subjective well-being. The science of happiness and a proposal for a national index. Am Psychol. 2000;55:34-43.

7. Hopwood P, Haviland J, Mills J, Sumo G, M Bliss J, START Trial Management Group. The impact of age and clinical factors on quality of life in early breast cancer: an analysis of 2208 women recruited to the UK START Trial (Standardisation of Breast Radiotherapy Trial). Breast. 2007;16:241-51.

8. Parmar V, Badwe RA, Hawaldar R, Rayabhattanavar S, Varghese A, Sharma R, et al. Validation of EORTC quality-of-life questionnaire in Indian women with operable breast cancer. Natl Med J India. 2005;18:172-7.

9. Schou I, Ekeberg $\varnothing$, Sandvik L, Hjermstad MJ, Ruland CM. Multiple predictors of health-related quality of life in early stage breast cancer. Data from a year follow-up study compared with the general population. Qual Life Res. 2005;14:1813-23.

10. Janz NK, Mujahid M, Lantz PM, Fagerlin A, Salem B, Morrow M, et al. Population-based study of the relationship of treatment and sociodemographics on quality of life for early stage breast cancer. Qual Life Res. 2005;14:1467-79.

11. Wenzel LB, Fairclough DL, Brady MJ, Cella D, Garrett KM, Kluhsman BC, et al. Age-related differences in the quality of life of breast carcinoma patients after treatment. Cancer. 1999;86:1768-74.

12. Aaronson NK, Ahmedzai S, Bergman B, Bullinger M, Cull A, Duez NJ, et al. The European Organization for Research and Treatment of Cancer QLQC30: a quality-of-life instrument for use in international clinical trials in oncology. J Natl Cancer Inst. 1993;85:365-76.

13. Sibhat SG, Fenta TG, Sander B, Gebretekle GB. Health-related quality of life and its predictors among patients with breast cancer at Tikur Anbessa Specialized Hospital, Addis Ababa, Ethiopia. Health Qual Life Outcomes. 2019;17:165.

14. Høyer M, Johansson B, Nordin K, Bergkvist L, Ahlgren J, Lidin-Lindqvist A, et al. Health-related quality of life among women with breast cancer - a population-based study. Acta Oncol. 2011;50:1015-26.

15. Hashemi SM, Balouchi A, Al-Mawali A, Rafiemanesh H, Rezaie-Keikhaie K, Bouya S, et al. Health-related quality of life of breast cancer patients in the Eastern Mediterranean region: a systematic review and meta-analysis. Breast Cancer Res Treat. 2019;174:585-96.

16. El Haidari R, Abbas LA, Nerich V, Anota A. Factors associated with health-related quality of life in women with breast cancer in the middle east: a systematic review. Cancers (Basel). 2020;12:696.

17. Elobaid YE, Aw TC, Grivna M, Nagelkerke N. Breast cancer screening awareness, knowledge, and practice among Arab women in the United Arab Emirates: a cross-sectional survey. PLoS One. 2014;9:e105783.

18. Elobaid YE. Breast cancer presentation delay among women in the united arab emirates. Dissertations. Paper 31.2014.

19. Bener A, Honein G, Carter AO, Da'ar Z, Miller C, Dunn EV. The determinants of breast cancer screening behavior: a focus group study of women in the United Arab Emirates. Oncol Nurs Forum. 2002;29:E91-8.

20. Fayers PM, Aaronson NK, Bjordal K, Groenvold M, Curran D, Bottomley A, et al. The EORTC QLQ-C30 scoring manual. Brussels: European Organisation for Research and Treatment of Cancer; 2001.

21. Sprangers MA, Groenvold M, Arraras JI, Franklin J, te Velde A, Muller M, et al. The European organization for research and treatment of cancer breast cancer-specific quality-of-life questionnaire module: first results from a three-country field study. J Clin Oncol. 1996;14:2756-68.

22. Fayers PM. Interpreting quality of life data: population-based reference data for the EORTC QLQ-C30. Eur J Cancer. 2001;37:1331-4.

23. Jassim GA, Whitford DL. Quality of life of Bahraini women with breast cancer: a cross sectional study. BMC Cancer. $2013 ; 13: 212$.

24. Nageeti TH, Elzahrany HR, Gabra AO, Obaid AA, Jastania RA. Quality of life assessment of breast cancer patients in Saudi Arabia. J Family Community Med. 2019;26:98-102.

25. Imran M, Al-Wassia R, Alkhayyat SS, Baig M, Al-Saati BA. Assessment of quality of life (QoL) in breast cancer patients by using EORTC QLQ-C30 and BR-23 questionnaires: A tertiary care center survey in the western region of Saudi Arabia. PLoS One. 2019;14:e0219093.

26. Alawadi SA, Ohaeri JU. Health - related quality of life of Kuwaiti women with breast cancer: a comparative study using the EORTC Quality of Life Questionnaire. BMC Cancer. 2009;9:222.

27. The healthcare system in the UAE. 2021. https://www.expatica.com/ae/healthcare/healthcare-basics/the-healthcare-system-in-the-united-arabemirates-71767/. Accessed 21 Oct 2021.

28. Jassim G, AlAnsari A. Reliability and validity of the Arabic Version of the EORTC QLQ-C30 and QLQ-BR23 questionnaires. Neuropsychiatr Dis Treat. 2020;16:3045-52.

Page 20/21 
29. Rahou BH, El Rhazi K, Ouasmani F, Nejjari C, Bekkali R, Montazeri A, et al. Quality of life in Arab women with breast cancer: a review of the literature. Health Qual Life Outcomes. 2016;14:64.

30. Almutairi KM, Mansour EA, Vinluan JM. A cross-sectional assessment of quality of life of breast cancer patients in Saudi Arabia. Public Health. 2016;136:117-25.

31. Paterson CL, Lengacher CA, Donovan KA, Kip KE, Tofthagen CS. Body image in younger breast cancer survivors: a systematic review. Cancer Nurs. 2016;39:E39-58.

\section{Supplementary Files}

This is a list of supplementary files associated with this preprint. Click to download.

- Appendix.docx 